\section{Der „einwilligungsunfähige“ Patient}

D er Patient kann nicht mehr gefragt werden: Was nun? Kann ein Patient nicht mehr - aus welchen Gründen auch immer - seinen Willen äußern, dann besteht „Einwilligungsunfähigkeit“. Diese zunächst festzustellen und dann angemessen und juristisch korrekt darauf zu reagieren, ist für Ärzte nicht immer leicht, zumal sich die gesetzlichen Grundlagen in den letzten Jahren mehrmals geändert haben. Daher erscheint der vorliegende Band auch schon in dritter, aktualisierter Auflage. Die gesetzliche
Regelung der Patientenverfügung vom 1.9.2009 ist darin berücksichtigt und brandaktuell.

Weitere Themen sind die Sterbebegleitung und Sterbehilfe sowie die zunehmende Bedeutung der palliativen Versorgung. Zur Erleichterung in der täglichen Praxis wurden für häufig vorkommende Fälle 18 Formularschreiben entwickelt. Schließlich enthält der Ratgeber auch Mustertexte für Patientenverfügungen und Betreuungsverfügungen. Kompakt und übersichtlich geschrieben ist dieses

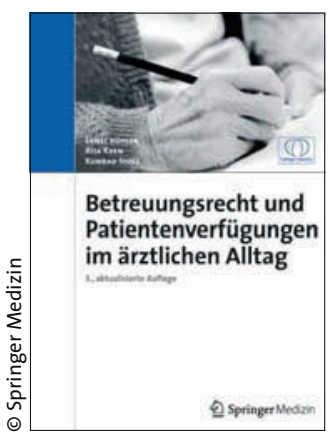

Bühler E, Kren R, Stolz K. Betreuungsrecht und Patientenverfügungen im ärztlichen Alltag. Springer Medizin, Urban \& Vogel, 2010, 100 S., 16,95 EUR, ISBN 9783-89935-261-O

Taschenbuch nicht für nur Ärzte, sondern auch für Angehörige von (womöglich bald) „Einwilligungsunfähigen" eine wertvolle Hilfe.

\title{
Kleine Geschichte der Hörhilfen
}

N ach seinem Standardwerk „Geschichte der Hörakustik" beschreibt Rainer Hüls „Eine kleine Geschichte der Hörhilfen“ mit dem Titel „Die Hand am Ohr". Das Buch ist ein unterhaltsamer und reich bebilderter Streifzug durch viele Jahrhunderte menschlicher Bemühungen, besser zu hören. Der weite Bogen spannt sich von den hörenden Perücken der Ägypter, Lichtschlangen und Hörvögeln über das Zeitalter der Hörrohre und den ersten fabrikmäßig herge-

stellten Hörgeräten bis zur Digitaltechnik und Nanotechnologie der Zukunft.

Prominente Persönlichkeiten aus allen Bereichen der menschlichen Gruppierung - stellvertretend seien Winston Churchill, Buzz Aldrin oder Sir Peter Ustinov genannt - werden mit liebevollem Augenzwinkern mit all ihren Hörproblemen dargestellt. Das Buch ist nicht nur eine interessante und kurzweilige Lektüre für Menschen mit Hörproblemen, es gibt auch dem HNO-Arzt

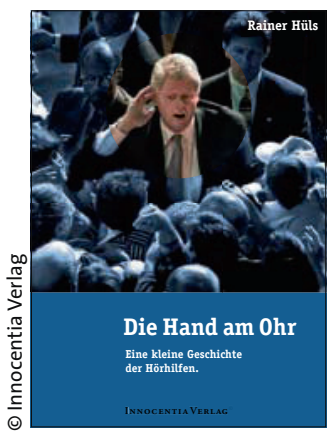

Hüls R. Die Hand am Ohr, Eine kleine Geschichte der Hörhilfen, Innocentia Verlag 2009, 208 S., 28 EUR, ISBN 978 3980810739

viele interessante Informationen zum Thema.

\section{Ein Muss für jeden Gutachter}

eder Richter am Sozialgericht, jeder Sachbearbeiter der Berufsgenossenschaft oder Sozialverwaltung und Rechtsanwalt hat ihn: den Schönberger/Mehrtens/Valentin. Er ist mit Sicherheit das meist zitierte Buch in Urteilen und Begründungen von Bescheiden über Berufskrankheiten. Für Nicht-Ärzte ist dieses Standardwerk auch der Brunnen des Verständnisses für beruflich bedingte $\mathrm{HNO}$ Erkrankungen: Lärmschwerhörigkeit, Riechstörungen, Kehlkopfkrebs, Adenokarzinome der Nasennebenhöhlen und Berufsunfälle. Für den gutachterlich interessierten HNO-Arzt ist dieses Buch auf jeden Fall ein Muss, da auch die komple- xen Zusammenhänge der Juristerei der Sozialgesetzgebung verständlich und leicht auffindbar abgehandelt werden. So wurde das gesamte Kapitel Lärmschwerhörigkeit neu überarbeitet, die aktuellsten Rahmenvereinbarungen über Hörgeräte, das neue Merkblatt der Bundesregierung zur Lärmschwerhörigkeit und die jüngste Rechtsprechung beschrieben. Die Besonderheiten der berufsbedingten Krebserkrankungen auf dem HNO-Fachgebiet werden ebenfalls umfassend dargelegt.

Vielleicht sieht man nicht sofort die Notwendigkeit, die Neuauflage zu kaufen, wenn man schon einen Schönberger/Mehrtens/Valentin hat; seien Sie aber

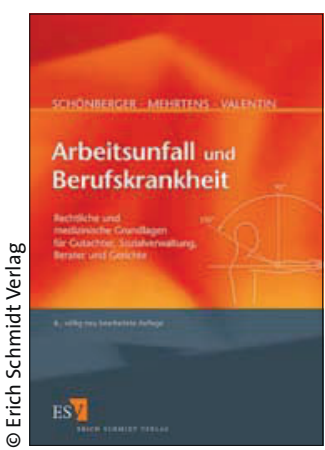

Schönberger A, Mehrtens $\mathrm{G}$, Valentin $\mathrm{H}$. Arbeitsunfall und Berufskrankheit. Erich Schmidt Verlag, 2010, 8 Auflage, 1.296 S., 178 EUR, ISBN 978-3503-11029-2

versichert, seit der letzten Auflage von 2003 hat sich viel zu viel geändert, als dass man auf die Neuauflage des treuen Ratgebers verzichten könnte, auch wenn man nur $\mathrm{ab}$ und zu ein Gutachten erstellt.

mi 